\title{
Parton distribution functions and hard QCD at HERA
}

\author{
Vladimir Chekelian* ${ }^{\dagger}$ \\ Max Planck Institute for Physics, Munich
}

The H1 and ZEUS collaborations at the electron-proton collider HERA have measured inclusive neutral and charged current deep inelastic scattering (DIS) cross sections over six orders of magnitude in negative four-momentum-transfer squared, $Q^{2}$, and Bjorken $x$. All these measurements were combined to obtain one coherent inclusive HERA data set. This set was used as the sole input to the QCD analysis resulting in the HERAPDF2.0 set of parton distribution functions. An inclusion of HERA data on jet and charm production to the QCD fit allowed a simultaneous determination of parton distributions and the strong coupling. An extension of earlier measurements on multijet production in DIS at high $Q^{2}$ to low values of $Q^{2}$ and to low values of the jet transverse momentum at high $Q^{2}$ is presented here as well as. A combination of the final H1 and ZEUS inclusive $D^{* \pm}$-meson production cross sections in DIS as a function of $Q^{2}$, inelasticity $y$ and $D^{* \pm}$ meson variables, and a measurement of isolated photon production in DIS accompanied by jets using the ZEUS detector are also presented. All these results are compared to QCD calculations.

38th International Conference on High Energy Physics

3-10 August 2016

Chicago, USA

\footnotetext{
* Speaker.

${ }^{\dagger}$ On behalf of the H1 and ZEUS Collaborations.
} 


\section{Introduction}

Deep inelastic scattering (DIS) of electrons ${ }^{1}$ on protons at the electron-proton collider HERA has been central to the exploration of proton structure, quark-gluon dynamics and the theory of strong interactions as described by perturbative Quantum Chromodynamics (pQCD). HERA was operated from 1992 to 2007 with an electron beam energy of $E_{e}=27.5 \mathrm{GeV}$ and proton beam energy of $E_{p}=920(820) \mathrm{GeV}$, corresponding to a centre-of-mass energy of $\sqrt{s} \simeq 320$ (300) GeV. At the end of data taking the proton beam energy was reduced to $E_{p}=575$ and $460 \mathrm{GeV}$, corresponding to $\sqrt{s} \simeq 251$ and $225 \mathrm{GeV}$. The $\mathrm{H} 1$ and ZEUS collaborations at HERA collected total integrated luminosities of approximately $500 \mathrm{pb}^{-1}$ each, shared about equally between $e^{+} p$ and $e^{-} p$ scattering.

All H1 and ZEUS inclusive neutral (NC) and charged current (CC) deep inelastic $e^{ \pm} p$ cross section measurements were combined [1] with a careful treatment of correlated systematic uncertainties and global normalisations of the data in the combination in order to obtain one coherent inclusive HERA data set. The dependencies of the combined NC and CC inclusive HERA cross sections on Bjorken $x$ and negative four-momentum-transfer squared, $Q^{2}$, were analysed in the pQCD framework to determine a new set of quark and gluon momentum distributions in the proton, denoted as HERAPDF2.0 [1], at leading (LO), next-to-leading (NLO) and next-to-next-toleading (NNLO) orders in the strong coupling $\alpha_{s}$. An inclusion of the HERA data on jet and charm production to the $\mathrm{pQCD}$ analysis allowed a simultaneous determination of the parton distribution functions (PDFs) and $\alpha_{s}$.

Recently, the H1 collaboration reported about an extension [2] of earlier measurements on inclusive jet, dijet and trijet production in DIS at high $Q^{2}$ [3] to low values of $Q^{2}$ and to low values of the jet transverse momentum at high $Q^{2}$. The jet cross section measurements were compared to NLO, approximate NNLO [4] and to full NNLO [5] QCD calculations.

The final $\mathrm{H} 1$ and ZEUS cross sections for inclusive $D^{* \pm}$-meson production in DIS were combined [6] in the visible phase space as a function of $Q^{2}$, inelasticity $y$, and as a function of $D^{* \pm}$ meson variables. The results were compared to perturbative NLO QCD predictions.

Further measurements of the production of isolated photons accompanied by jets in DIS [7] were accomplished using the ZEUS detector. The measured cross sections were compared to Monte Carlo (MC) simulation and to QCD calculations based on the $k_{T}$ factorisation method.

\section{Combination of inclusive $\mathrm{NC}$ and $\mathrm{CC}$ data at HERA and HERAPDF2.0}

In order to combine all inclusive $\mathrm{NC}$ and $\mathrm{CC}$ deep inelastic cross section measurements published by $\mathrm{H} 1$ and ZEUS, they were translated onto common $x, Q^{2}$ grids and averaged [1]. In total 41 data sets from H1 and ZEUS entered into this combination. The 2927 published cross sections from these 41 data sets were combined to become 1307 combined cross-section measurements for values of $Q^{2}$ between $Q^{2}=0.045 \mathrm{GeV}^{2}$ and $Q^{2}=50000 \mathrm{GeV}^{2}$, and for values of $x$ between $x=6 \times 10^{-7}$ and $x=0.65$. For the resulting 1620 degrees of freedom, a $\chi^{2}=1687$ was obtained, indicating consistency of the input data. The total uncertainties for $\mathrm{NC}$ are below $1.5 \%$ over the $Q^{2}$ range of $3 \leq Q^{2} \leq 500 \mathrm{GeV}^{2}$ and below $3 \%$ up to $Q^{2}=3000 \mathrm{GeV}^{2}$.

\footnotetext{
${ }^{1}$ The word "electron" in the paper refers to both electrons and positrons, unless otherwise stated.
} 


\section{H1 and ZEUS}

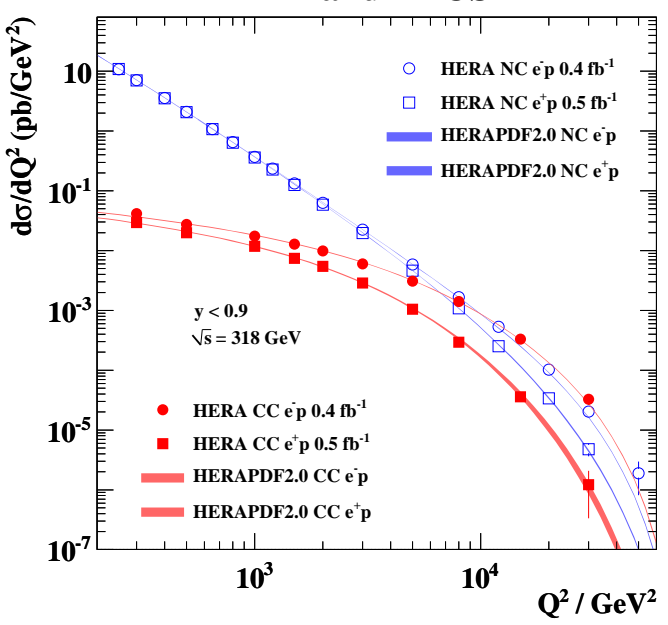

H1 and ZEUS

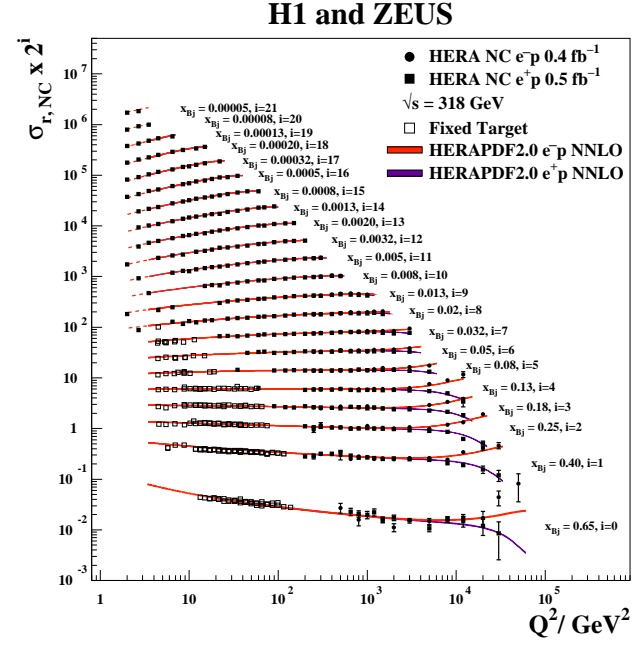

Figure 1: Left: The combined HERA NC and CC $e^{-} p$ and $e^{+} p$ cross sections, $\mathrm{d} \sigma / \mathrm{d} Q^{2}$, together with predictions from HERAPDF2.0 NLO. Right: The combined HERA data for the inclusive $\mathrm{NC} e^{+} p$ and $e^{-} p$ reduced cross sections together with fixed-target data and the predictions of HERAPDF2.0 NNLO.

The combined $\mathrm{NC}$ and $\mathrm{CC}$ cross sections for $e^{-} p$ and $e^{+} p$ scattering, integrated to obtain the single-differential cross sections $\mathrm{d} \sigma / \mathrm{d} Q^{2}$, are shown in Fig. 1 (left). Whereas the NC cross sections are three orders of magnitude larger at low $Q^{2} \approx 100 \mathrm{GeV}^{2}$, where they are dominated by virtual photon exchange, the $\mathrm{NC}$ and $\mathrm{CC}$ cross sections become similar in magnitude at $Q^{2} \approx 10000 \mathrm{GeV}^{2}$, i.e. at around the mass-scale squared of the electroweak bosons, demonstrating the success of electroweak unification in the Standard Model.

The dominant contribution to the $\mathrm{NC}$ cross section is due to the proton structure function $F_{2}$. Therefore, the combined reduced NC cross sections shown in Fig. 1 (right) reveal the main properties of this proton structure function. At $x \simeq 0.1$ they are independent of $Q^{2}$ demonstrating scaling of $F_{2}$ in this region. At higher and lower values of $x$ one observes scaling violations of $F_{2}$ due to emission of gluons from quarks and splitting of gluons into quark-antiquark pairs, respectively. From the difference of the combined $e^{-} p$ and $e^{+} p$ NC cross sections at $Q^{2} \geq 1000 \mathrm{GeV}^{2}$, the proton structure function $x F_{3}^{\gamma Z}$ was measured as well.

The combined inclusive $e^{ \pm} p$ NC and CC cross section data were used as the sole input in the pQCD analysis, resulting in the PDF set called HERAPDF2.0 [1]. In this analysis the predictions obtained by solving the DGLAP evolution equations at LO, NLO and NNLO in the $\overline{\mathrm{MS}}$ scheme were fitted to the combined inclusive HERA data. The DGLAP equations yield the PDFs at all scales $\mu_{\mathrm{f}}^{2}$ and $x$, if they are provided as functions of $x$ at some starting scale. In variable-flavournumber schemes, this scale has to be below the charm-quark mass parameter, $M_{c}$, squared. It was chosen to be $1.9 \mathrm{GeV}^{2}$. The renormalisation and factorisation scales were taken to be $Q^{2}$. The heavy-quark coefficient functions were calculated in the general-mass variable-flavour-number scheme called RTOPT [8] for the NC structure functions. For the CC structure functions, the zeromass approximation was used, since all HERA CC data have $Q^{2} \gg M_{b}^{2}$, where $M_{b}$ is the beautyquark mass parameter in the calculation. The value of $M_{c}$ and $M_{b}$ were chosen after performing 

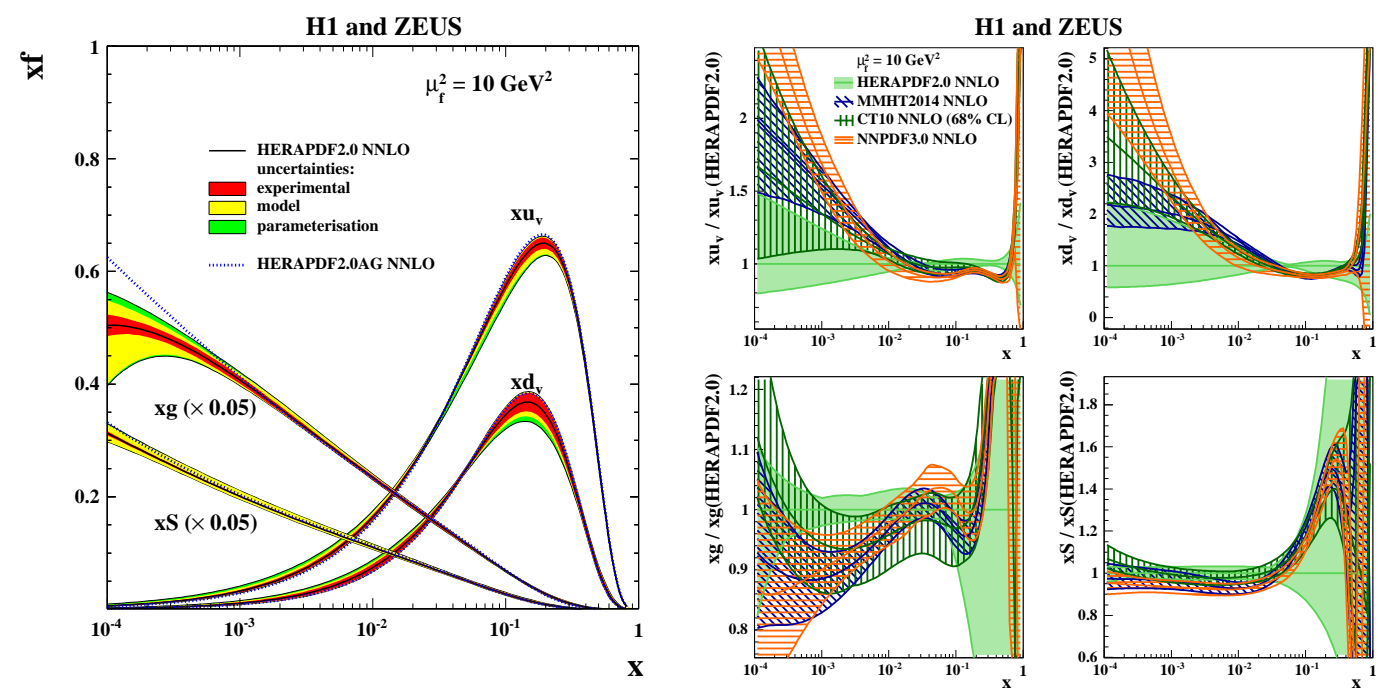

Figure 2: The parton distribution functions $x u_{v}, x d_{v}, x S=2 x(\bar{U}+\bar{D})$ and $x g$ at $\mu_{\mathrm{f}}^{2}=10 \mathrm{GeV}^{2}$. Left: HERAPDF2.0 NNLO with the experimental, model and parameterisation uncertainty bands and HERAPDF2.0AG NNLO represented by the dotted lines; $x S$ and $x g$ are scaled down by a factor of 20. Right: The ratios of the NNLO PDFs of HERAPDF2.0, MMHT2014, CT10 and NNPDF3.0 with corresponding uncertainty bands to the central PDF values of HERAPDF2.0 NNLO.

$\chi^{2}$ scans of NLO and NNLO pQCD fits to the combined inclusive data together with the HERA combined charm data [9] or with data on beauty production from H1 [10] and ZEUS [11]. The value of the strong coupling constant at the Z-boson mass scale, $\alpha_{s}\left(M_{Z}\right)$, was taken to be $\alpha_{s}\left(M_{Z}\right)=$ $0.118(0.130)$ for the fits at NLO and NNLO (LO).

In the approach of HERAPDF, the PDFs of the proton, $x f$, are generically parameterised at the starting scale as $x f(x)=A x^{B}(1-x)^{C}\left(1+D x+E x^{2}\right)$. The PDFs parameterised are the gluon distribution, $x g$, the valence-quark distributions, $x u_{v}, x d_{v}$, and the $u$-type and $d$-type anti-quark distributions, $x \bar{U}, x \bar{D}$. The relations $x \bar{U}=x \bar{u}$ and $x \bar{D}=x \bar{d}+x \bar{s}$ are assumed at the starting scale. In the gluon distribution, $x g$, an additional term of the form $A_{g}^{\prime} x^{B_{g}^{\prime}}(1-x)^{C_{g}^{\prime}}$ was subtracted at NLO and NNLO to allow more flexibility for the gluon distribution. The fits without this term are marked by adding "AG" (alternative gluon) to the fit notation. In total 14 free parameterisation parameters were determined in the central fits.

To safely remain in the kinematic region where $\mathrm{pQCD}$ is expected to be applicable, only cross sections for $Q^{2}$ starting from $Q_{\min }^{2}=3.5 \mathrm{GeV}^{2}$ were used in the analysis. In this kinematic region, target-mass corrections and large- $x$ higher-twist corrections are expected to be negligible. Experimental uncertainties were determined using the Hessian method with the criterion $\Delta \chi^{2}=1$, justified by the consistency of the input HERA data. In addition to experimental uncertainties, model and parameterisation uncertainties were also considered.

Using the settings discussed above, the HERAPDF2.0 sets of PDFs were determined at NNLO, NLO and LO. A summary of HERAPDF2.0 NNLO is shown in Fig. 2 (left) at the scale $\mu_{\mathrm{f}}^{2}=$ $10 \mathrm{GeV}^{2}$. The experimental, model and parameterisation uncertainties are shown separately. At NNLO, the gluon distribution of HERAPDF2.0 ceases to rise at low $x$ in contrast to HERAPDF2.0AG NNLO obtained using the alternative, positively defined, gluon parameterisation. 


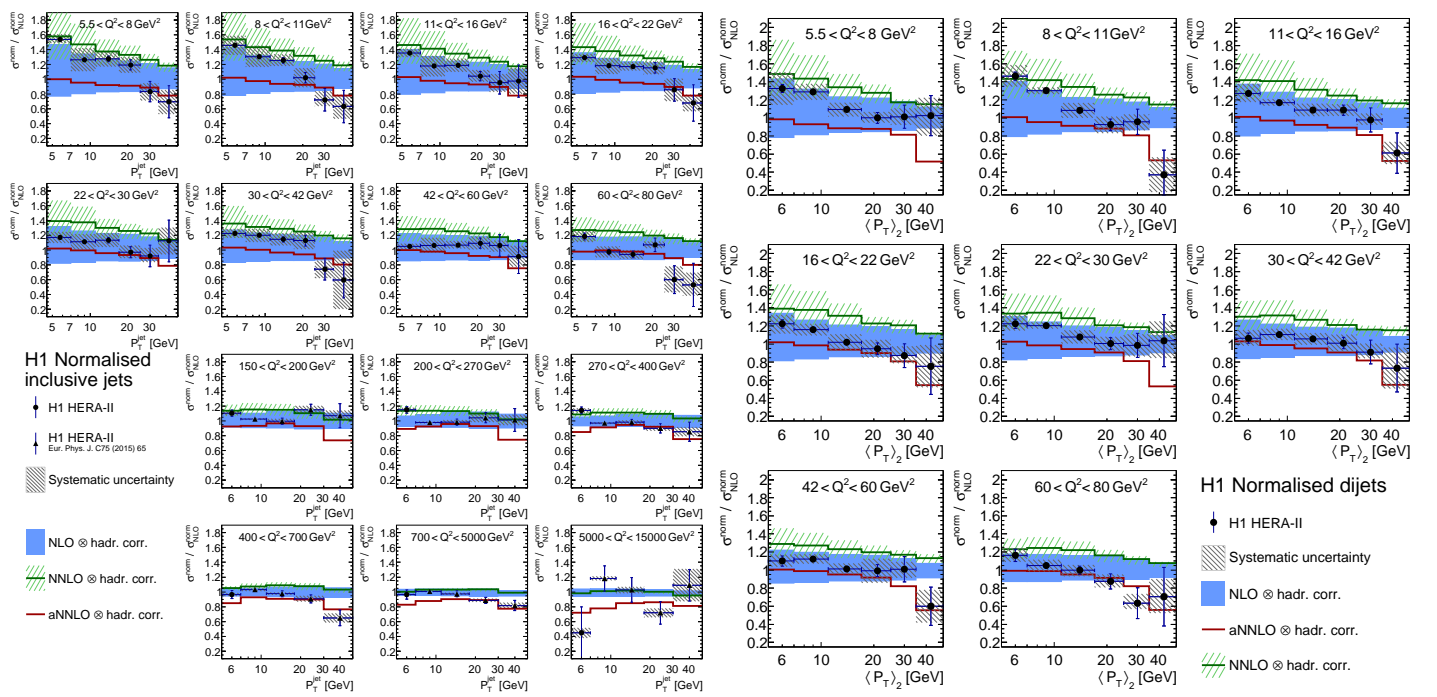

Figure 3: The normalised double differential cross section measurements for inclusive jets (left) and dijets (right) divided by the NLO calculations corrected for hadronisation. The aNNLO and NNLO predictions are shown as well.

The PDFs of HERAPDF2.0 NNLO are compared in Fig. 2 (right) to MMHT2014 [12] using the same heavy-flavour scheme, i.e. RTOPT, to CT10 [13] with a heavy-flavour-scheme based on ACOT, and to NNPDF3.0 [14] with the FONLL scheme. All PDFs are compatible taking into account full uncertainties. The variants of the QCD fit, HERAPDF2.0FF3A and HERAPDF2.0FF3B, were obtained using fixed-flavour-number schemes, which are similar to $A B M 11 \mathrm{FF}(3 \mathrm{~N})$ [15] and NNPDF3.0 FF(3N) [14], respectively. Another variant HERAPDF2.0HiQ2, with a larger value of $Q_{\min }^{2}, Q_{\min }^{2}=10 \mathrm{GeV}^{2}$, was obtained. An extension of the analysis by including HERA data on jet and charm [9] production, denoted as HERAPDF2.0Jets, made a simultaneous determination of parton distributions and the strong coupling constant at the scale $M_{Z}$ possible. The $\chi^{2}$ and the resulting PDFs of the HERAPDF2.0Jets fit are very close to the values for the fit with fixed $\alpha_{s}\left(M_{Z}\right)=0.118$. The result, $\alpha_{s}\left(M_{Z}\right)=0.1183 \pm 0.0009(\exp ) \pm 0.0005$ (model $\left./ \mathrm{param}.\right) \pm$ 0.0012 (hadron.) ${ }_{-0.0030}^{+0.0037}$ (scale), is compatible with the world average [16] and competitive with other determinations at NLO.

\section{Multijets in DIS and theory predictions at NNLO}

In the QCD fit leading to HERAPDF2.0Jets [1], mentioned in the previous section, the H1 measurements of the inclusive jet, dijet and trijet cross sections at high $Q^{2}$ [3] were included. Recently, the H1 collaboration reported an extension [2] of these measurements to low $Q^{2}, 5.5<$ $Q^{2}<80 \mathrm{GeV}^{2}$, and the transverse momentum $4.5<P_{T}^{j e t}<50 \mathrm{GeV}$. Additionally, inclusive jets at low $P_{T}^{\text {jet }}, 5<P_{T}^{\text {jet }}<7 \mathrm{GeV}$, and high $Q^{2}, 150<Q^{2}<15000 \mathrm{GeV}^{2}$, were measured as well. The jet cross sections were divided by the inclusive NC DIS cross sections in the corresponding $Q^{2}$-bins to obtain normalised jet cross sections.

Jets were reconstructed using the inclusive $k_{T}$ algorithm in the Breit frame and were required to have a minimum transverse momentum of $4 \mathrm{GeV}$. The precision of the jet cross section measure- 
ments is in the range of $6 \%$ to $20 \%$ in the central parts of the phase space. The results for inclusive jet and dijet double differential cross sections as functions of $Q^{2}$ and $P_{T}$ or of $Q^{2}$ and the average transverse momentum of two jets in the dijets events, $\left\langle P_{T}^{\text {jet }}\right\rangle_{2}$, are shown in Fig. 3 (left and right) divided by the pQCD calculations at NLO corrected for hadronisation effects. New theoretical calculations in the threshold resummation formalism with 2-loop contributions (aNNLO) [4] and new predictions in full NNLO [5] perturbative QCD are also shown. For the theory calculations the PDFs of NNPDF3.0 at NLO and NNLO were used. The predictions in NNLO perturbative QCD improve the descriptions for inclusive jet and dijet cross sections compared to NLO predictions, and give an overall good description of the data. The sensitivity of the jet data to the extraction of the strong coupling constant and to the running of $\alpha_{s}$ was studied in a fit to NLO predictions. The $\alpha_{s}\left(M_{Z}\right)$ value from the $\mathrm{H} 1$ normalised jet data in the range $5.5<Q^{2}<15000 \mathrm{GeV}^{2}$ was found to be $\alpha_{s}\left(M_{Z}\right)=0.1173 \pm 0.0004(\exp )_{-0.0045}^{+0.0053}$ (theory), demonstrating the high experimental precision of the data.

\section{ep $\rightarrow \mathrm{eD}^{\star \pm} \mathrm{X}$}

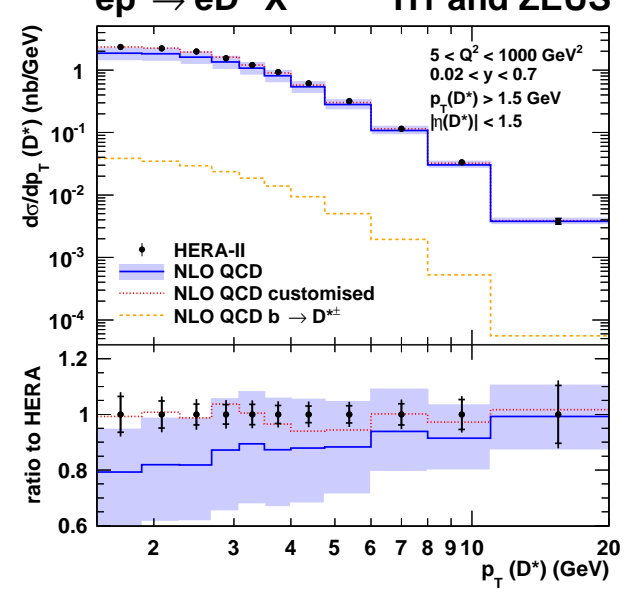

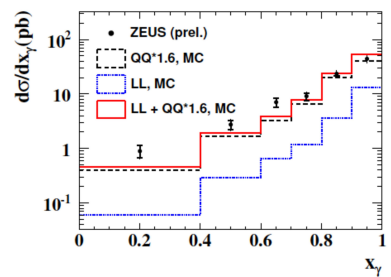
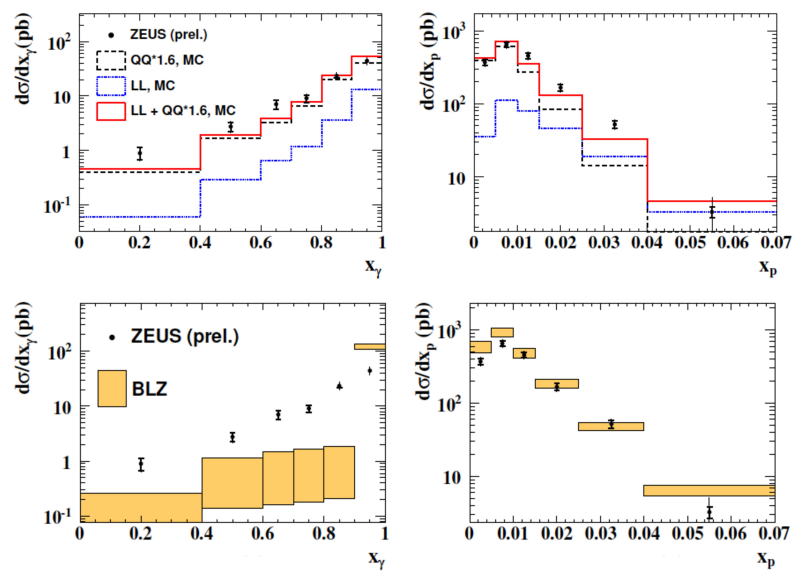

Figure 4: Left: The combined HERA $D^{* \pm}$-production cross section as a function of $p_{T}\left(D^{*}\right)$ together with the NLO prediction and with a customised NLO calculation. The ratios to the central values of the combined cross sections are shown in the bottom part. Right: Production cross sections of isolated photons accompanied by jets in DIS as a function of $x_{\gamma}$ and $x_{p}$, compared to the reweighted MC calculations (top) and to the BLZ theoretical predictions (bottom).

\section{Combination of visible $D^{* \pm}$ cross sections in DIS at HERA}

Different techniques have been applied at HERA to measure open-charm production in DIS. After extrapolation from the visible to the full phase space, most of these data have been combined [9] at the level of the reduced cross-sections and have provided a consistent determination of the charm contribution to the proton structure functions. These data have been used in the HERAPDF2.0 analysis to determine the charm-quark mass parameter $M_{c}$ [1]. The extrapolation procedure required theoretical assumptions and was restricted to inclusive DIS variables $Q^{2}$ and inelasticity $y$ only. Alternatively, the measured cross sections can be combined directly in the visible phase space of the measurements. In this case, dependences on the theoretical input are minimised and the charm production mechanism can be explored in terms of other variables. 
The visible $D^{* \pm}$-production cross sections from H1 and ZEUS at the centre-of-mass energy $\sqrt{s} \simeq 320 \mathrm{GeV}$ were combined such that one consistent HERA data set is obtained [6]. The combination procedure accounted for all correlations and yields a significant reduction of the overall uncertainties of the measurements. The combined HERA cross sections were measured as a function of $Q^{2}$ and $y$, and of the $D^{* \pm}$-meson's transverse momentum, $p_{T}\left(D^{*}\right)$, pseudorapidity, $\eta\left(D^{*}\right)$, and inelasticity, $z\left(D^{*}\right)$. The combined data were compared to NLO pQCD predictions. The comparison for $p_{T}\left(D^{*}\right)$ is shown in Fig. 4 (left). The data reach an overall precision of about 5\% over a large fraction of the measured phase space, while the typical theoretical uncertainty ranges from $30 \%$ at low $Q^{2}$ to $10 \%$ at high $Q^{2}$. The predictions describe the data well within their uncertainties. Higher order calculations would be helpful to reduce the theory uncertainty to a level more comparable with the data precision.

\section{Prompt photon production accompanied by jets in DIS}

The production cross section of an isolated photon in DIS, accompanied by at least one additional jet, was measured [7] with the ZEUS detector at HERA using an integrated luminosity of $326 \mathrm{pb}^{-1}$. The jets were reconstructed using the $k_{T}$-clustering algorithm with the $\mathrm{R}$ parameter set to 1.0. An isolated photon was detected exploiting the fine segmentation of the ZEUS uraniumscintillator calorimeter in the beam direction. The single-differential cross sections were measured as a function of the fraction of the incoming photon energy, $x_{\gamma}$, that is given to the photon and the jet, the fraction of proton energy, $x_{p}$, taken by the parton that interacts with the photon, the azimuthal angle, $\Delta \phi$, and the pseudorapidity difference, $\Delta \eta$, between the prompt photon and the jet, and the azimuthal angle, $\Delta \phi^{e, \gamma}$, and the pseudorapidity difference, $\Delta \eta^{e, \gamma}$, between the prompt photon and the scattered electron. The distributions in $x_{\gamma}$ and $x_{p}$ are shown in Fig. 4 (right). On top the measurements are compared to the predictions for the sum of the photon emission from the incoming beam or scattered electron (LL) and from a quark as a part of the hard process (QQ). The LL contribution was simulated using Djangoh, and the expected QQ contribution from Pythia was multiplied by a factor of 1.6. This model provides a good description of the data. On the bottom, the prompt photon data are compared to the theory calculations based on the $k_{T}$ factorisation method used by BLZ [17]. No hadronic corrections were applied, nevertheless the theory describes the overall shape of the data adequately, except for $x_{\gamma}$ and $\Delta \phi$.

\section{Summary}

The H1 and ZEUS collaborations measured inclusive $e^{ \pm} p$ scattering cross sections at HERA from 1994 to 2007, collecting a total integrated luminosity of about $1 \mathrm{fb}^{-1}$ at four different centreof-mass energies, with two different detectors changing and improving over time. All inclusive data were combined to create one consistent set of $\mathrm{NC}$ and $\mathrm{CC}$ cross-section measurements for $e^{ \pm} p$ scattering, spanning six orders of magnitude in both negative four-momentum-transfer squared, $Q^{2}$, and Bjorken $x$. They are the most precise measurements ever published for ep scattering over such a large kinematic range. The combined inclusive HERA cross sections were used as input to a QCD analysis. The resulting parton distribution functions are denoted HERAPDF2.0 and are available at LO, NLO and NNLO using a variable-flavour-number scheme. Fits were also 
performed exploring an alternative, positively defined, gluon parameterisation, different values of $Q_{\min }^{2}$ and various fixed-flavour-number schemes. A measurement of the strong coupling constant was performed in a perturbative QCD fit, for which the inclusive cross sections were augmented with selected jet- and charm-production cross section data at HERA.

The H1 collaboration reported about an extension of earlier measurements on inclusive jet, dijet and trijet production in DIS at high $Q^{2}$ to low values of $Q^{2}, 5.5<Q^{2}<80 \mathrm{GeV}^{2}$, and to low $P_{T}^{j e t}, 5<P_{T}^{j e t}<7 \mathrm{GeV}$, at $150<Q^{2}<15000 \mathrm{GeV}^{2}$. The jet production cross sections were compared to NLO, approximate NNLO and to full NNLO QCD calculations. The NNLO predictions improve the descriptions for inclusive jet and dijet cross sections compared to NLO predictions, and provide an overall good description of the data.

Measurements of $D^{ \pm}$-production cross sections in deep inelastic ep scattering by the $\mathrm{H} 1$ and ZEUS collaborations were combined at the level of visible cross sections, accounting for their systematic correlations. The data sets were found to be consistent, and the combined data have significantly reduced uncertainties. QCD predictions in NLO describe the data well.

The production of isolated photons accompanied by jets has been measured in deep inelastic scattering with the ZEUS detector. The present results complement the earlier ZEUS results, which studied different variables of interest. The results are in agreement with Pythia after a re-scaling has been applied and can be used to make further improvements in the QCD calculations.

\section{References}

[1] H. Abramowicz et al. [H1 and ZEUS Collab.], Eur. Phys. J. C 75, 580 (2015), [arXiv:1506.06042].

[2] H1 Collaboration, DESY-16-200, [arXiv:1611.03421].

[3] V. Andreev et al., [H1 Collaboration], Eur. Phys. J. C 75, 2 (2015), [arXiv:1406.4709].

[4] T. Biekötter, M. Klasen and G. Kramer, Phys. Rev. D 92, 074037 (2015), [arXiv:1508.07153].

[5] J. Currie, T. Gehrmann and J. Niehues, Phys. Rev. Lett. 117, 042001 (2016), [arXiv:1606.03991].

[6] H. Abramowicz et al. [H1 and ZEUS Collaborations], JHEP 09, 149 (2015), [arXiv:1503.06042].

[7] ZEUS Collaboration, ZEUS-prel-16-001.

[8] R. S. Thorne and R. G. Roberts, Phys. Rev. D 57, 6871 (1998), [hep-ph/9709442];

R. S. Thorne, Phys. Rev. D 73, 054019 (2006), [hep-ph/0601245];

R. S. Thorne, Phys. Rev. D 86, 074017 (2012), [arXiv:1201.6180].

[9] F. Aaron et al. [H1 and ZEUS Collaborations], Eur. Phys. J. C 73, 2311 (2013), [arXiv:1211.1182].

[10] F. Aaron et al. [H1 Collaboration], Eur. Phys. J. C 65, 89 (2009), [arXiv:0907.2643].

[11] H. Abramowicz et al. [ZEUS Collaboration], JHEP 1409, 127 (2014), [arXiv:1405.6915].

[12] L. Harland-Lang, A. D.Martin, P. Motylinski, and R. Thorne (2014), [arXiv:1412.3989].

[13] M. Guzzi et al., Phys. Rev. D 89, 033009 (2014), [arXiv:1302.6246].

[14] R. D. Ball et al. [NNPDF Collaboration], JHEP 1004, 040 (2015), [arXiv:1410.8849v2].

[15] S. Alekhin et al., Phys. Rev. D 86, 054009 (2012), [arXiv:1202.2281].

[16] J. Beringer et al.. (Particle Data Group), Phys. Rev. D 86, 010001 (2012).

[17] S. Baranov, A. Lipatov and N. Zotov, Phys. Rev. D 81, 094034 (2010). 